\title{
Specialized drug delivery systems
}

\author{
Hoo-Kyun Choi ${ }^{1}$
}

Published online: 4 July 2016

(C) The Korean Society of Pharmaceutical Sciences and Technology 2016

It is my great pleasure and honor to guest edit this special issue of Journal of Pharmaceutical Investigation, 2016. All the authors who participated in the issue are prominent scientists in each field. The theme of this issue is "Specialized drug delivery systems." We may have had more than enough of this topic. Nonetheless, the reason I chose the topic was that there are still many areas that have not been sufficiently explored and that require to be updated due to a significant amount of advances and interest in the field. I will briefly introduce the contents of this issue.

Liposomes have been used for more than 50 years to deliver various drugs; however, their use has been limited by various drawbacks of liposome itself including physical stability, problems during blood circulation, and complications in mass production. Various attempts, including surface modifications and conjugations, were successfully utilized to overcome drawbacks and liposome-based products have been commercialized for targeted delivery of various cancer drugs based on EPR effect. More recently, lipid-based carriers, including liposomes and emulsions, have drawn great deal of attention as efficient and safer antigen delivery systems. Although peptide-based subunit vaccines and DNA vaccines are known to be safer vaccines than traditional vaccines, subunit or DNA vaccines still suffer from limited immunogenicity and short-lived immune responses. After considerable investigations on adjuvants and antigen delivery systems, lipid-based carriers were chosen due to high biocompatibility, ease of

Hoo-Kyun Choi

hgchoi@chosun.ac.kr

1 College of Pharmacy, Chosun University, Gwangju 61452, Republic of Korea manipulation, and efficient delivery to target cells. Many different kinds of lipids, including neutral, cationic, and anionic lipids, and various surface-modification methods were investigated to improve the delivery systems. As a result, two vaccine products, Epaxal ${ }^{\mathrm{TM}}$ and Inflexal $\mathrm{V}^{\mathrm{TM}}$, are commercialized using liposome, and several others are being developed for commercialization. Cationic lipidbased nanoparticles have been investigated as a carrier for gene delivery. Gene delivery systems mainly use two different vectors, i.e., viral vector and non-viral vector. These two vectors have their own pros and cons. Viral vector is the more efficient vector of these delivery systems; however, it has a safety issue. While non-viral vector is safer to use, it has a low delivery efficiency. Adenovirus-derived vector is one of the most common vectors used in clinical trials, and numerous efforts have been made to overcome the current limitations of adenovirus-derived vectors. One of the most promising efforts is the electrostatic complexation of adenoviral vectors with cationic lipid-based particles.

Nanoparticles have been one of the main subjects in the area of drug delivery. The most frequent target disease of nanoparticular drug delivery system has been cancer. It has been well known that combination chemotherapy minimizes the incidence of resistance owing to multiple molecular mechanisms. Recent development in nanotechnology has enabled controlled and targeted delivery of combination chemotherapeutics. Many different kinds of nanoparticles are prepared in this field including liposome, polymeric micelle, polymeric nanoparticle, polymer-drug conjugate, dendrimer, and mesoporous silica nanoparticle. One of various materials used to prepare nanoparticles is albumin. Albumin has various advantages over synthetic polymers. Albumin is known to be biocompatible and non- 
toxic, and is viewed as one of the most useful and versatile carriers. In addition, it can extend circulation half-lives of peptide and protein drugs when they are properly linked. Albumin is one of the best materials to prepare nanoparticles due to proper physicochemical stability, targetability, and chemical functionality. Some of the most frequently used methods to prepare albumin nanoparticles are desolvation, nanoparticle albumin-bound technology, and selfassembly. The most frequent route of administration used to administer nanoparticles is IV injection. Nanotechnology also has been utilized in oral drug delivery systems. Nanomedicines for oral delivery may be classified as lipidbased nanomedicine, polymer-based nanomedicine, and nanosuspension. Oral administration of nanomedicine has more complicated barriers that are hard to overcome. Extensive investigations are required and are being conducted to solve those problems.

Another promising approach to treat cancer is antibodydrug conjugate. It combines the specificity of monoclonal antibodies with the cytotoxicity of anti-tumor drugs. Recently, two antibody-drug conjugate products, i.e., brentuximab vedotin (Adcetris) and trastuzumab emtansine (Kadcyla), have received FDA approval, and there are many other candidates in clinical trials for the treatment of various cancers. One of the problems with the current antibody-drug conjugate is the drug-to-antibody ratio (heterogenic). Various efforts, including engineered cysteines, insertion of unnatural amino acids, and enzymatic ligation, are being made to prepare homogeneous antibody-drug conjugates.

Stents are extensively used in the recanalization of vascular and nonvascular occlusions and prevent future stenosis. One of the major problems associated of using stents is restenosis. Drug-eluting stent is widely used to prevent restenosis. Polymeric materials play a very important role in the preparation of drug-eluting stent. Proper selection of drug, physicochemical properties of the drug, required duration, and release profile of the drug must be taken into account. Both natural polymers and synthetic polymers have been used. Various coating methods are available including dip coating, spray coating, electrospinning, and rolling coating.

It is interesting that montmorillonite is recently being considered as an excipient to develop drug delivery system. It is a multifunctional clay mineral and a major component of bentonite. It can be swelled and has high adsorption capacity. It has been shown that it can increase drug entrapment, maintain sustained release, and enhance dissolution rate and bioavailability of hydrophobic drugs. It may also be used to form composites with various polymeric drug delivery systems. Another material that is the focus of interest in the drug-delivery area is probiotics. Probiotics are live microorganisms and have been used for a long time to confer health benefits. The key issue in the delivery of probiotics is its viability until it reaches target, intestine, since it is vulnerable to several environmental factors such as temperature and $\mathrm{pH}$. The successful delivery of probiotics has been drawing significant attention of drug delivery scientists these days.

In conclusion, this issue of Journal of Pharmaceutical Investigation will provide readers with many different aspects of current drug delivery systems and insight into future of drug delivery sciences. 